\title{
Hydroxychloroquine and chloroquine for treatment of coronavirus disease 19 (COVID-19): a systematic review and meta-analysis of randomized and non-randomized controlled trials
}

\author{
Risa Ebina-Shibuya ${ }^{1}$, Ho Namkoong ${ }^{2}$, Nobuyuki Horita ${ }^{3}$, Hideaki Kato ${ }^{4}$, Yu Hara ${ }^{3}$, Nobuaki Kobayashi ${ }^{3}$, \\ Takeshi Kaneko ${ }^{3}$ \\ ${ }^{1}$ Laboratory of Molecular Immunology, Immunology Center, National Heart, Lung, and Blood Institute, National Institutes of Health, Bethesda, \\ MD, USA; ${ }^{2}$ Laboratory of Clinical Immunology and Microbiology, National Institute of Allergy and Infectious Diseases, National Institutes of \\ Health, Bethesda, MD, USA; ${ }^{3}$ Department of Pulmonology, Yokohama City University Graduate School of Medicine, Yokohama, Japan; Infection \\ Prevention and Control Department, Yokohama City University Hospital, Yokohama, Japan \\ Contributions: (I) Conception and design: R Ebina-Shibuya, Y Hara, N Kobayashi; (II) Administrative support: T Kaneko; (III) Provision of study \\ materials or patients: R Ebina-Shibuya, H Namkoong, N Horita; (IV) Collection and assembly of data: R Ebina-Shibuya, H Namkoong, N Horita; (V) \\ Data analysis and interpretation: All authors; (VI) Manuscript writing: All authors; (VII) Final approval of manuscript: All authors. \\ Correspondence to: Nobuyuki Horita, MD, PhD. Department of Pulmonology, Yokohama City University Graduate School of Medicine, 3-9, Fukuura, \\ Kanazawa, Yokohama, Japan. Email: horitano@yokohama-cu.ac.jp.
}

Background: Although the US government approved hydroxychloroquine (HCQ) and chloroquine (CQ) for hospitalized coronavirus disease 19 (COVID-19) patients, some studies denied efficacy of HCQ and CQ. We aimed to evaluate HCQ/CQ treatment for COVID-19.

Methods: Five databases were searched on April 15, 2020, without publication date restriction. We followed both Preferred Reporting Items for Systematic Reviews and Meta-analyses and Meta-analysis of Observational Studies in Epidemiology statement reporting recommendations. A random-model metaanalysis was conducted to pool odds ratio (OR) and hazard ratio (HR). The quality of evidence for each outcome and the final recommendation was assessed using the GRADE guidelines of the American College of Chest Physicians.

Results: We identified four randomized controlled trials (RCTs) and four observational studies with 2,063 COVID-19 cases. All-cause mortality was not affected by the administration of HCQ/CQ [OR: 1.05, 95\% confidence interval (CI): 0.53-2.09, $\mathrm{P}=0.89$ ]. No improvement of viral clearance was found neither by timeto-event analysis (HR: 1.19, 95\% CI: 0.74-1.94, P=0.47) nor frequency on day 7 (OR: 1.47, 95\% CI: $0.33-$ 6.63, $\mathrm{P}=0.62$ ). HCQ/CQ treatment increased the risk of the any adverse event with OR of 3.56 (95\% CI: $1.62-7.83, \mathrm{P}=0.002)$.

Conclusions: HCQ/CQ failed to decrease the all-cause mortality (very low quality evidence) and did not improve viral clearance (low or very low quality evidence) but increased the risk of any adverse event (moderate quality evidence). Routine administration of HCQ/CQ for COVID-19 patients is not recommended (weak recommendation, Grade 2C).

Keywords: Virus diseases; aminoquinolines; evidence-based medicine; drug approval; policy making; coronavirus disease 19 (COVID-19)

Submitted May 24, 2020. Accepted for publication Nov 05, 2020.

doi: $10.21037 /$ jtd-20-2022

View this article at: http://dx.doi.org/10.21037/jtd-20-2022 


\section{Introduction}

As of April 22, 2020, more than 2.5 million people have been diagnosed with coronavirus disease 2019 (COVID-19). It is caused by a novel coronavirus, severe acute respiratory syndrome coronavirus (SARS-CoV-2), which causes severe pneumonia leading to fatal respiratory failure (1). Several existing drugs including the antimalarials, chloroquine (CQ) and hydroxychloroquine (HCQ), have been extensively studied as possible treatment candidates. A Chinese expert panel recommended CQ for COVID-19 cases in February 2020, primarily based on in vitro data and clinical data from CQ-treated patients who were infected with other pathogens (2). In vitro, CQ potently blocked SARS$\mathrm{CoV}-2$ infection at low micromolar concentrations with a high selectivity index (3). In mid-March 2020, a small size non-randomized clinical trial conducted by Gautret et al. revealed that HCQ treated COVID-19 patients experienced more rapid viral load reduction than did those without HCQ (4). At the end of March 2020, the US Food and Drug Administration (FDA) authorized clinicians to prescribe HCQ/CQ for hospitalized patients without any evidence generated from a randomized controlled trial (RCT) $(5,6)$.

Presently perception is that HCQ and CQ, which are safe, old, and cheap drugs with antiviral efficacy, have clinical applicability to SARS-CoV-2. Nonetheless, their efficacy remains unclear due to the lack of conclusive data from RCTs. Moreover, the narrow margin of safety of CQ occasionally causes severe side effects including central nervous disorders, ocular manifestations, and arrhythmia due to QT prolongation (7). Although HCQ is safer than CQ, uncontrolled use of these drugs can lead to serious toxicities. Another concern is the possible shortage of CQ and HCQ for well-established treatment indications, which would adversely affect people with rheumatological disorders, including systemic lupus erythematosus (SLE), rheumatoid arthritis (RA), and primary Sjögren syndrome (8). Several randomized and non-randomized controlled trials with conflicting results have been reported from China and France since mid-March (4,9-14). Based on the results of these studies, we performed a systematic review and metaanalysis to provide deeper insights about the suitability of HCQ/CQ for the treatment of COVID-19. We present the following article in accordance with the PRIMSA reporting checklist (available at http://dx.doi.org/10.21037/jtd-202022).

\section{Methods}

\section{Overview}

Patient informed consent and institutional ethics review board approval were not applicable since our review did not directly deal with patients and clinical data. The protocol has been registered on the University hospital Medical Information Network Center Clinical Trial Registry (UMIN-CTR, Japan) as UMIN000040188 (15). Our systematic review followed Preferred Reporting Items for Systematic Reviews and Meta-analyses and Meta-analysis of Observational Studies in Epidemiology statement $(16,17)$.

\section{Study search}

We searched for candidate articles using PubMed, Cochrane CENTRAL, EMBASE, and Web of Science Core Collection on April 15th, 2020. To detect non-peer reviewed recent articles, medRxiv was also searched. Search strategy is presented elsewhere (Appendix 1).

\section{Publication type}

RCTs and observational studies that compared treatment with and without HCQ/CQ written in any language were included. A retrospective study that compared treatment outcome by HCQ/CQ regimen and non-HCQ/CQ regimen was added. Our analysis did not exclude conference abstracts and non-peer reviewed articles. Single-arm studies and case reports were not included because these studies do not have control arms. For studies that included three or more arms, we extracted the HCQ/CQ arm and the most appropriate counterpart arm.

\section{Patient}

Only studies with confirmed COVID-19 diagnosis patient were included. Although we did not pre-specify diagnosis method and patient disposition, only hospitalized patients with PCR confirmed diagnoses were recruited in the studies that we finally evaluated.

\section{Treatment}

A patient in the HCQ/CQ arm should be treated with HCQ or CQ at any dosage for any duration. Concomitant administration of azithromycin (AZM) was permitted. 


\section{Outcome}

Protocol specified primary outcomes were all-cause mortality and viral clearance. Viral clearance was chosen as a primary outcome because enhanced viral clearance with HCQ has been reported from a French non-randomized trial, which contributed to US FDA approval of HCQ and CQ (4-6).

Secondary outcomes included time to discharge, symptom alleviation, CT image improvement, disease progression defined as a composite outcome with death and intensive care unit (ICU) admission, any adverse event, and serious adverse event.

For viral clearance and CT improvement, we extracted both time-to-event hazard ratio (HR) and event occurrence at day 7 . In the absence of day 7 data, data on day 6 or 8 were used instead.

\section{Subgroup analysis}

Subgroup analyses for all outcomes from RCTs and observational studies were performed.

\section{Study selection and data extraction}

Articles found in PubMed, Embase, Web of Science, and Cochrane were screened based on title and abstract information, scrutinized through full-article reading performed independently by two review authors (RES \& $\mathrm{NH}$ ), who also extracted necessary data independently. Similarly, articles from medRxiv were processed by two review authors ( $\mathrm{HN} \& \mathrm{NH})$.

For observational studies, multiple variate-adjusted value or propensity score weighted value was used when available. Study characteristics such as study design, treatment regimen, and trial size and outcome such as mortality and viral clearance were extracted. We did not try to contact authors of the original studies because we aimed an expedited reporting.

\section{Quality assessment}

Because the widely used Cochrane Risk of Bias tool is designed for RCTs, we assessed the risk of bias of each study with the concept of Cochrane Risk of Bias tool and described the methodological weaknesses that may lead to high risk of bias (18).

\section{Statistics}

Dichotomous outcomes were compared using the MantelHaenszel odds ratio (OR). When one or more cells in the two-by-two contingency were null, 0.5 was added to all the cells. HRs were pooled after logarithmic transformation. The inverse variance method was used where appropriate. When data were obtainable, intention-to-treat analysis was preferred except for adverse event analysis, for which safety analysis sets should be used. Kaplan-Meier curves were read using Parmer's method as deemed necessary (19).

A random-effect model meta-analysis was performed using Review Manager version 5 (Cochrane, London, UK). Publication bias was not assessed due to the number of studies, which was less than 10 .

\section{Quality of evidence and recommendation}

Quality of evidence of each outcome was judged as high, moderate, low, or very low as per the GRADE guideline (20) Final grade of recommendation was decided based on the system used by the American College of Chest Physicians (21).

\section{Results}

\section{Study selection}

We found 319 articles by electronic search and two additional articles from hand search. Of 321 articles that met the preliminary criteria, 53, 234, and 26 were excluded through removal of duplication, title/abstract screening, and full-article scrutinizing, respectively (Figure 1). Ultimately, we selected eight articles for our quantitative synthesis (4,9-14,22,23) (Table 1, Figure 1).

\section{Characteristics of included studies}

The eight research papers consisted of four RCT and four observational studies, five were from China, and two were from France, one was from USA; seven were written in English language, and one was written in Chinese language (Table 1). One article we found in medRxiv was published in peer reviewed article after the submission of our first draft $(12,22)$. Numbers of patients included in each study ranged from 22 to 1,438 with a median of 130 , a total of 2,063. Four adopted HCQ regimens, two used CQ regimens, one used an HCQ + AZM regimen, and one adopted a factorial 


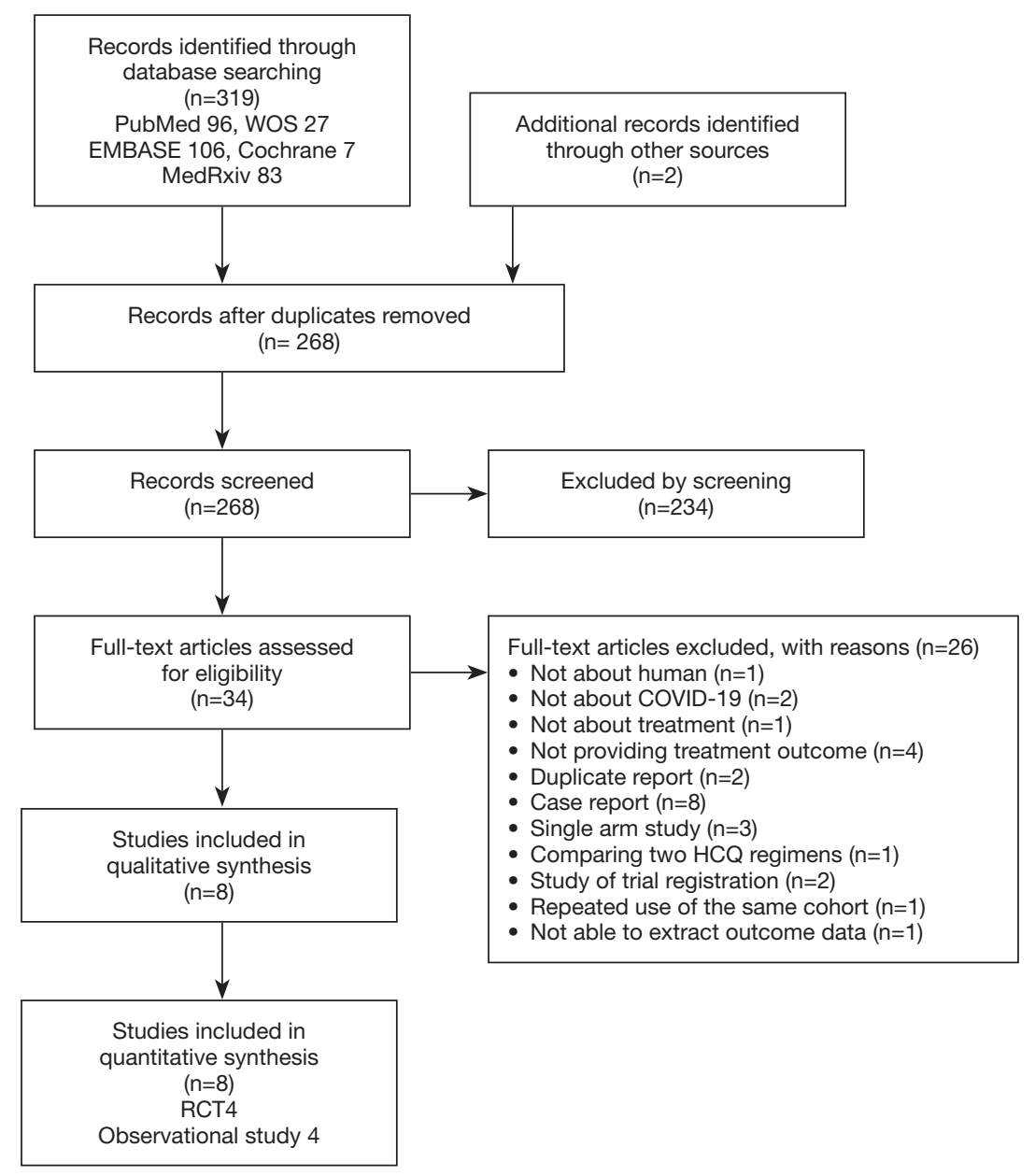

Figure 1 PRISMA flow diagram. WOS, Web of Science; HCQ, hydroxychloroquine; COVID-19, coronavirus disease 19.

design combining HCQ and AZM. Doses of HCQ ranged from 400 to $1,200 \mathrm{mg} /$ day. Two retrospective observational study did not specify the HCQ/CQ dosage. One study administered Lopinavir/Ritonavir as a counterpart of HCQ/CQ, while the other seven did not employ any specific anti-viral treatment. According to five reports that described disease severity, most patients had asymptomatic, mild, or moderate disease with three exceptions: Tang et al. and Huang et al. randomized two and eight severe cases $(11,12,22)$, respectively; and approximately $16 \%$ of COVID-19 cases reported by of Chen et al. were serious or critical (Table 1). Each study had several methodological weaknesses which led to high risks of bias as shown in Table 1 .

\section{All-cause mortality}

A study by Rosenberg recorded by far the largest number of death cases. This observational study compared four arms, HCQ + AZM, HCQ alone, AZM alone, and neither of them. Therefore, we derived two comparison from this study: "HCQ + AZM versus AZM alone" and "HCQ alone versus neither of them". All-cause mortality was not affected by the administration of HCQ/CQ [OR: 1.05, 95\% confidence interval $(\mathrm{CI}): 0.53-2.09, \mathrm{P}=0.89 ; \mathrm{I}^{2}=31 \%$, P for heterogeneity 0.18 ; Figure $2 A]$.

\section{Viral clearance}

According to time to event analysis, HCQ/CQ treatment did not facilitate the viral clearance (HR: 1.19, 95\% CI: 0.74 1.94, $\mathrm{P}=0.47 ; \mathrm{I}^{2}=57 \%, \mathrm{P}$ for heterogeneity 0.07 ; Figure $2 B$ ).

Data for viral clearance at day 7 were reported in four articles. Sixty-nine (55\%) out of $126 \mathrm{HCQ} / \mathrm{CQ}$ treated patients and 67 (57\%) out of 118 patients in the control arm 


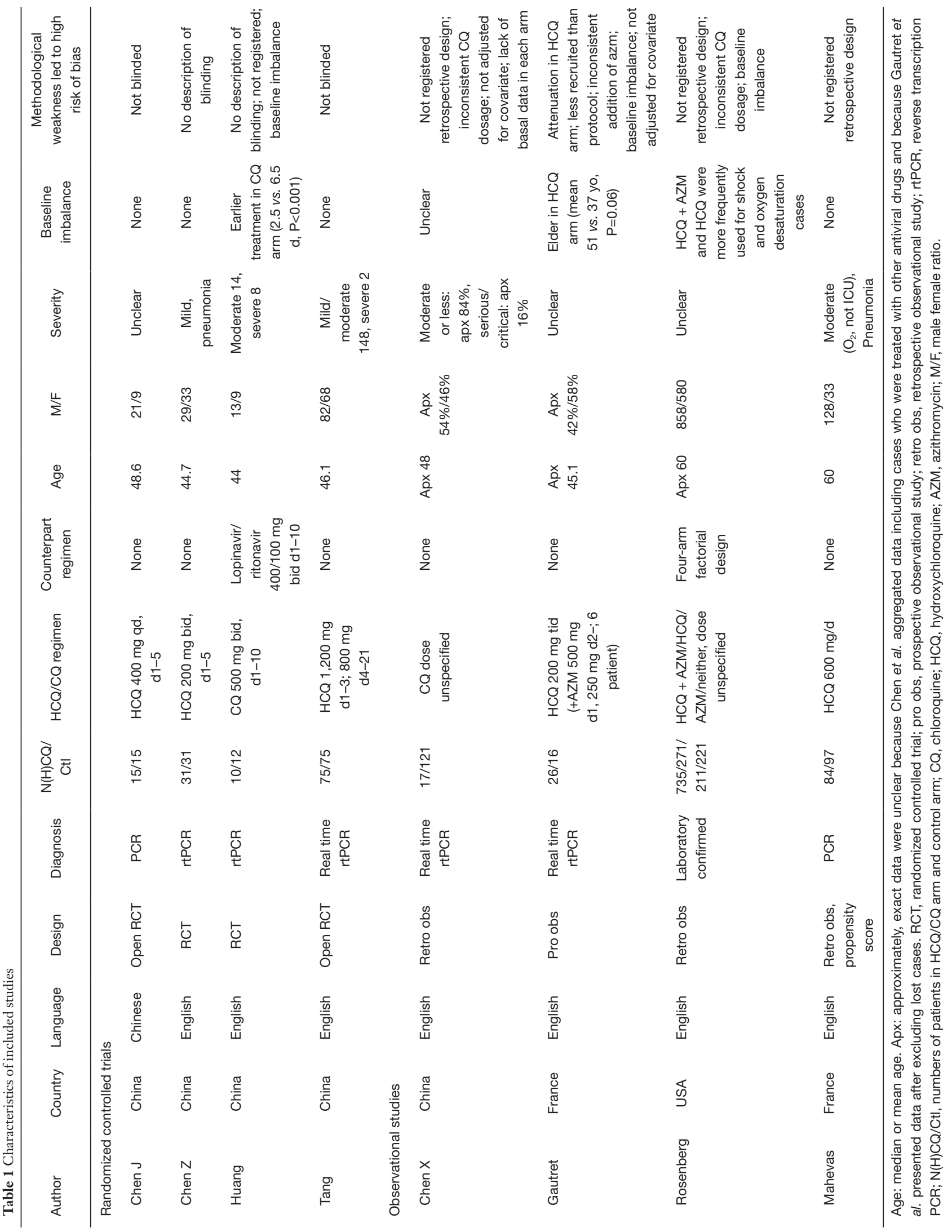



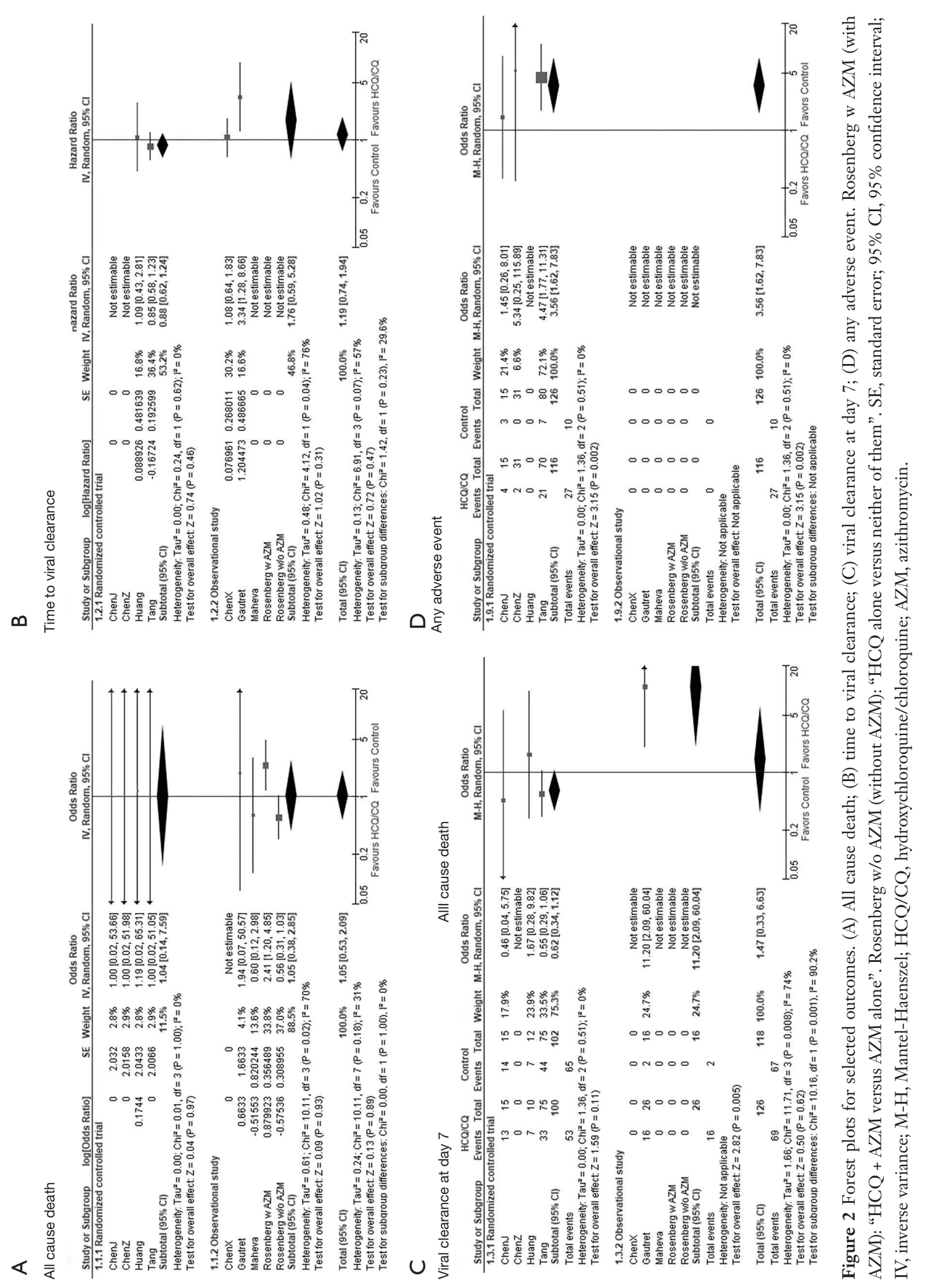
achieved viral clearance at day 7 . There was no difference in the frequency of day 7 viral clearance between the arms (OR: 1.47, 95\% CI: 0.33-6.63, P=0.62; Figure 2C). Substantial overall heterogeneity $\left(\mathrm{I}^{2}=74 \%, \mathrm{P}\right.$ for heterogeneity $\left.<0.001\right)$ may be attributed to the study design $\left(\mathrm{I}^{2}=90.2 \%, \mathrm{P}\right.$ for subgroup difference $<0.001$ ).

\section{Time to discharge}

A random-model meta-analysis with two studies consisted of 160 patients showed that $\mathrm{HCQ} / \mathrm{CQ}$ resulted in a tendency toward faster discharge with an HR of 2.29 (95\% CI: $0.72-7.27, \mathrm{P}=0.16 ; \mathrm{I}^{2}=73 \%, \mathrm{P}$ for heterogeneity 0.06 ; Figure S1).

\section{Time to symptom alleviation}

An RCT by Tang et al. is the only trial that provided a HR for time to symptom alleviation. However, this trial found no difference for symptom alleviation (HR: 1.01, 95\% CI: 0.59-1.75, $\mathrm{P}=0.97$; Figure S2)

\section{Time to CT image improvement}

Huang et al. reported a small size RCT with 22 patients, which compared CQ $500 \mathrm{mg}$ po twice daily for 10 days and oral Lopinavir and Ritonavir. CQ regimen was associated with a tendency toward faster CT image improvement (HR: 2.42, 95\% CI: 0.83-7.05, $\mathrm{P}=0.10$; Figure S3).

$\mathrm{HCQ} / \mathrm{CQ}$ administration was not associated with frequency of CT image improvement at day 7 (OR: 1.22, 95\% CI: $0.28-5.29, \mathrm{P}=0.79 ; \mathrm{I}^{2}=54 \%, \mathrm{P}$ for heterogeneity 0.11 ; Figure S4)

\section{Disease progression}

Two observational studies provided data on the composited outcome of death and ICU admission. The pooled OR from these two studies with 223 patients was 1.41 (95\% CI: $0.28-$ $7.05, \mathrm{P}=0.68 ; \mathrm{I}^{2}=37 \%$, P for heterogeneity 0.21 ; Figure $\mathrm{S} 5$ )

\section{Adverse event}

Random-model meta-analyses of three RCTs consisting of 242 cases indicated that HCQ/CQ treatment increased the risk of any adverse event with OR of 3.56 (95\% CI: 1.62-7.83, $\mathrm{P}=0.002)$ and without heterogeneity $\left(\mathrm{I}^{2}=0, \mathrm{P}\right.$ for heterogeneity 0.51 ; Figure $1 D$ ). However, administration of HCQ/CQ did not lead to a significant increase of severe adverse event (OR: 2.11, 95\% CI: 0.27-16.68, P=0.48; $\mathrm{I}^{2}=0 \%, \mathrm{P}$ for heterogeneity 0.74 ; Figure S6).

\section{Quality of evidence and recommendation}

The findings from the current systematic review and metaanalysis accompanied with graded evidence level of each outcome are summarized in Table 2 (20).

Therefore, with this body of evidence, it is not recommended to administrate $\mathrm{HCQ} / \mathrm{CQ}$ for COVID-19 cases (weak recommendation, low or very low quality evidence, Grade 2C) (21).

\section{Sensitivity analysis focusing peer reviewed articles}

Although this analysis was not specified in the protocol, some may want to know excluding non-peer reviewed articles may alter the primary endpoint results. Analysis based on peer reviewed article only did not change the conclusion as shown in Figure S7.

\section{Discussion}

We have performed a systematic review and meta-analysis, quantitatively integrating four RCTs and four observational studies involving 2,063 patients, to elucidate how HCQ/ CQ affects COVID-19 clinical outcomes. The first peerreviewed clinical study that evaluated efficacy of $\mathrm{HCQ}$ with a non-HCQ arm was reported by Gautret et al. from France (4) on March 20, 2020. At day 6 post-inclusion, $70 \%$ of $\mathrm{HCQ}$-treated patients achieved viral clearance compared to $12.5 \%$ in the control group $(\mathrm{P}=0.001)$ (4). This drastic therapeutic impact of HCQ facilitated US FDA approval of HQC and QC to treat SARS-CoV-2 infection $(5,6)$. However, three subsequent RCTs did not reproduce this $(9,11,12)$. Our analysis did not confirm any clinical benefit including SARS-CoV-2 clearance by HCQ/CQ administration this treatment increased any adverse events (OR: 3.56, 95\% CI: 1.62-7.83, P=0.002; no heterogeneity; moderate quality of evidence) (Table 2, Figure 2B,C). Our result does not conflict with recent RCT by Boulware (24), which demonstrated postexposure prophylactic HCQ did not prevent illness compatible with COVID-19 or confirmed infection (24).

HCQ and CQ are drugs with a wide range of antiinflammatory, immunomodulatory, anti-malarial, antiviral, and anti-tumor effects (25). Although their molecular 
Table 2 Summary of finding table

\begin{tabular}{|c|c|c|c|c|c|c|c|}
\hline & Study & $\mathrm{Pt}$ & OR or HR $(95 \% \mathrm{Cl})$ & $\mathrm{P}$ & $\begin{array}{c}\mathrm{I}^{2}, \mathrm{P} \text { for } \\
\text { heterogeneity }\end{array}$ & $\begin{array}{l}\text { Quality of } \\
\text { evidence }\end{array}$ & $\begin{array}{c}\text { Reason for quality } \\
\text { of evidence down } \\
\text { grading }\end{array}$ \\
\hline Death from any cause & 7 & 1,925 & OR: 1.05 (0.53-2.09) & 0.89 & $31 \%, 0.18$ & Very low & Non-RCT, RoB \\
\hline Time to viral clearance & 4 & 352 & HR: 1.19 (0.74-1.94) & 0.47 & $57 \%, 0.07$ & Low & Non-RCT, RoB \\
\hline \multicolumn{8}{|l|}{ Secondary outcome } \\
\hline Time to discharge & 2 & 160 & HR: 2.29 (0.72-7.27) & 0.16 & $73 \%, 0.06$ & Very low & $\begin{array}{l}\text { Non-RCT, RoB, } \\
\text { imprecision }\end{array}$ \\
\hline CT image improvement at day 7 & 3 & 114 & OR: 1.22 (0.28-5.29) & 0.79 & $54 \%, 0.11$ & Very low & RoB, imprecision \\
\hline $\begin{array}{l}\text { Disease progression (death or } \\
\text { ICU admission) }\end{array}$ & 2 & 223 & OR: $1.41(0.28-7.05)$ & 0.68 & $37 \%, 0.21$ & Very low & $\begin{array}{l}\text { Non-RCT, RoB, } \\
\text { imprecision }\end{array}$ \\
\hline Any adverse event & 3 & 242 & OR: 3.56 (1.62-7.83) & 0.002 & $0 \%, 0.51$ & Moderate & RoB \\
\hline Serious adverse event & 3 & 242 & OR: $2.11(0.27-16.68)$ & 0.48 & $0 \%, 0.74$ & Low & RoB, imprecision \\
\hline
\end{tabular}

Quality of evidence was graded into four ranks: high, moderate, low, and very low according to GRADE guidelines. Non-RCT: including observational study; RoB: high risk of bias; imprecision: wide $95 \% \mathrm{Cl}$ of the pooled value. Inconsistency: significant heterogeneity. OR, odds ratio; HR, hazard ratio; $95 \% \mathrm{CI}, 95 \%$ confidence interval; $\mathrm{RCT}$, randomized controlled trial; ICU, intensive care unit.

mechanisms are not clear, inhibition of autophagy and Tolllike receptor function have been reported. CQ has been shown to have in vitro activity against multiple RNA viruses including SARS-CoV-1 (26). It has also been noted that HCQ and CQ may inhibit sialic acid synthesis, protein glycosylation, and the cellular interactions involved in viral attachment and invasion $(27,28)$.

From our meta-analysis, in addition to the lack of a convincing therapeutic effect, there was a significant increase in any adverse events, but not for the severe adverse events (Figure 2D). HCQ and CQ are generally well-tolerated medications, but clinicians and patients should be aware of the serious adverse events that can occur, even during short courses of the treatment. Notorious harmful events include prolongation of the QT interval, which can cause arrhythmic death. While HCQ alone did not increased death compared to "neither of them" arm in the Rosenberg's observation, HCQ + AZM led to increased death compared to AZM alone. Abnormal electrocardiogram finding was noted in as many as $27.1 \%$ of patients with HCQ + AZM (Table 2) (23). Particular attention should be paid to arrhythmia high-risk patients and the concomitant use of AZM because both AZM and HCQ independently cause QT prolongation (29). A recent phase IIb RCT in Brazil that compared high- and low-dose HCQ for COVID-19 cases was prematurely halted because frequent QT prolongation and increased lethality were observed in the high-dose arm of $12 \mathrm{~g} /$ day for 10 days (30). Transient gastrointestinal adverse events including diarrhea, abdominal pain, and abnormal liver function were also commonly observed in RCTs (9-12). Other side effects including rash, itchiness, and retinal damage have also been reported (9-12,31).

Both HCQ and CQ have been employed successfully for the treatment of SLE and RA over 70 years (32). All patients with SLE of any degree and type of disease activity should be treated with HCQ or CQ, unless these agents are contraindicated (33). Specially, HCQ has a good reputation for controlling erythematosus. A prolonged shortage of HCQ and CQ, which would like to be produced by widespread administration of HCQ and CQ for COVID-19, will deprive people with SLE and RA of an 
effective, well-tolerated, and low-cost drugs. Patients and rheumatologists are terrified that they might lose access to their key drugs, further threatening disease progression and quality of living (8).

Currently, we do not have any treatment option or vaccination verified by large-scale trials; despite the need for swift action to stop this catastrophic pandemic. Many people tend to jump at any attractive information, despite the very low quality of clinical evidence provided by smallscale observational studies or in vitro experiments. The global COVID-19 pandemic has also exerted pressure on clinicians and regulatory authorities to act quickly to provide effective drugs to patients. Some groups have argued that the authorities should promptly approve a drug without any RCT data $(5,6,34)$. Based on limited in vitro and anecdotal clinical data from case series, HCQ and CQ are currently recommended for the treatment of hospitalized COVID-19 patients in several countries (6). For example, a Chinese expert panel recommended oral CQ, $500 \mathrm{mg}$ twice per day for 10 days for patients diagnosed with mild, moderate, and severe COVID-19 cases without scrutinizing the clinical evidence (2). The US FDA approved the prescription of HCQ and CQ for hospitalized patients and is facilitating the availability of HCQ and CQ during the COVID-19 pandemic to treat as many patients as possible who are not eligible for a clinical trial $(5,6)$. Despite warnings from scientific advisers, the FDA adopted this policy based on a single, small observational study showing that HCQ treated COVID-19 patients experienced prompt viral load clearance (4). This faster viral clearance in HCQ arm was not confirmed by subsequent RCTs (Figure 2B,C). These unprecedented policy-making processes are contrary to the foundation of evidence-based medicine (35). Non-evidence based medical policy decisions put the patient safety and drug reviewing system at risk, which requires "substantial evidence" of safety and efficacy based on well-controlled and well-designed trials before a drug can be marketed (34).

We would like to comment on the possible advantages from HCQ/CQ treatment. Administration of CQ was associated with trend toward shorter hospital stay (HR: 2.29, 95\% CI: 0.72-7.27, $\mathrm{P}=0.16)(11,13)$ (Figure S1). Our analysis was not able to completely refute this possible therapeutic merit. If quicker recovery can be achieved by HCQ or CQ, this would greatly improve the crisis of medical resource shortage. Future RCTs may confirm this clinical benefit of HCQ.

Limitations of our analysis should also be mentioned. First, all the included studies had considerable methodological weaknesses leading to certain high risk of bias, primarily due to the non-randomized study designs and a lack of blinding. Second, pooled statistics for many outcomes were compromised by the small number of studies and patients. Therefore, the quality of evidence for each outcome is not high (Table 2). Third, the majority of COVID-19 patients in our analysis had asymptomatic, mild, or moderate disease; thus, external validity of our analysis for severe and critical cases is weak. Finally, our systematic review did not evaluate combination of HCQ/CQ with other antiviral medications. Despite these considerable limitations, we offer our analysis as a response to the urgent need for the summarized data for HCQ/CQ treatment on COVID-19.

In conclusion, based on the available evidence, the routine administration of HCQ/CQ for COVID-19 patients is not recommended (weak recommendation, Grade 2C). This treatment is associated with an increased risk of adverse events without any conclusive clinical benefit including faster viral clearance. Although the currently available evidence is not yet conclusive, numerous relevant RCTs have been listed on the trial registration website (36). We hope that these trials and additional systematic reviews will update the evidence regarding this important topic.

\section{Acknowledgments}

Funding: None.

\section{Footnote}

Reporting Checklist: The authors have completed the PRIMSA reporting checklist. Available at http://dx.doi. org/10.21037/jtd-20-2022

Peer Review File: Available at http://dx.doi.org/10.21037/jtd20-2022

Conflicts of Interest: All authors have completed the ICMJE uniform disclosure form (available at http://dx.doi. org/10.21037/jtd-20-2022). TK received a lecture fee from Sanofi in Japan. The other authors have no conflicts of interest to declare.

Ethical Statement: The authors are accountable for all aspects of the work in ensuring that questions related to the accuracy or integrity of any part of the work are appropriately investigated and resolved. 
Open Access Statement: This is an Open Access article distributed in accordance with the Creative Commons Attribution-NonCommercial-NoDerivs 4.0 International License (CC BY-NC-ND 4.0), which permits the noncommercial replication and distribution of the article with the strict proviso that no changes or edits are made and the original work is properly cited (including links to both the formal publication through the relevant DOI and the license). See: https://creativecommons.org/licenses/by-nc-nd/4.0/.

\section{References}

1. Zhu N, Zhang D, Wang W, et al. A Novel Coronavirus from Patients with Pneumonia in China, 2019. N Engl J Med 2020;382:727-33.

2. Multicenter Collaboration Group of Department of Science and Technology of Guangdong Province and Health Commission of Guangdong Province for Chloroquine in the Treatment of Novel Coronavirus Pneumonia. Expert consensus on chloroquine phosphate for the treatment of novel coronavirus pneumonia. Zhonghua Jie He He Hu Xi Za Zhi 2020;43:E019.

3. Wang M, Cao R, Zhang L, et al. Remdesivir and chloroquine effectively inhibit the recently emerged novel coronavirus (2019-nCoV) in vitro. Cell Res 2020;30:269-71.

4. Gautret P, Lagier JC, Parola P, et al. Hydroxychloroquine and azithromycin as a treatment of COVID-19: results of an open-label non-randomized clinical trial. Int J Antimicrob Agents 2020;56:105949.

5. Lenzer J. Covid-19: US gives emergency approval to hydroxychloroquine despite lack of evidence. BMJ 2020;369:m1335.

6. Hinton DM. Food and Drug Administration. FDA Emergency use authorization (EUA) of chloroquine and hydroxychloroquine. 28 Mar 2020. Available online: https://www.fda.gov/media/136534/download (Accessed Apr 20, 2020).

7. Frisk-Holmberg M, Bergqvist Y, Englund U. Chloroquine intoxication. Br J Clin Pharmacol 1983;15:502-3.

8. Jakhar D, Kaur I. Potential of chloroquine and hydroxychloroquine to treat COVID-19 causes fears of shortages among people with systemic lupus erythematosus. Nat Med 2020;26:632.

9. Chen J, Liu D, Liu L, et al. A pilot study of hydroxychloroquine in treatment of patients with common coronavirus disease-19 (COVID-19). J Zhejiang Univ (Med Sci) 2020. doi: 10.3785/j.issn.1008-9292.2020.03.03.

10. Chen Z, Hu J, Zhang Z, et al. Efficacy of hydroxychloroquine in patients with COVID-19: results of a randomized clinical trial. medRxiv 2020. doi: 10.1101/2020.03.22.20040758.

11. Huang $M$, Tang T, Pang $\mathrm{P}$, et al. Treating COVID-19 with chloroquine. J Mol Cell Biol 2020;12:322-5.

12. Tang W, Cao Z, Han, M, et al. Hydroxychloroquine in patients with COVID-19: an open-label, randomized, controlled trial. medRxiv 2020. doi: 10.1101/2020.04.10.20060558.

13. Chen X, Zhang Y, Zhu B. Associations of clinical characteristics and antiviral drugs with viral RNA clearance in patients with COVID-19 in Guangzhou, China: a retrospective cohort study. medRxiv 2020. doi: 10.1101/2020.04.09.20058941.

14. Mahevas M, Tran VT, Roumier M, et al. No evidence of clinical efficacy of hydroxychloroquine in patients hospitalized for COVID-19 infection with oxygen requirement: results of a study using routinely collected data to emulate a target trial. medRxiv 2020. doi: 10.1101/2020.04.10.20060699.

15. University hospital Medical Information Network Center Clinical Trial Registry. Available online: https://www.umin. ac.jp/icdr/index.html (Accessed April 2020).

16. Moher D, Liberati A, Tetzlaff J, et al. Preferred reporting items for systematic reviews and meta-analyses: the PRISMA statement. BMJ 2009;339:b2535.

17. Stroup DF, Berlin JA, Morton SC, et al. Meta-analysis of observational studies in epidemiology: a proposal for reporting. Meta-analysis Of Observational Studies in Epidemiology (MOOSE) group. JAMA 2000;283:2008-12.

18. Higgins JP, Altman DG, Gotzsche PC, et al. The Cochrane Collaboration's tool for assessing risk of bias in randomised trials. BMJ 2011;343:d5928.

19. Parmar MK, Torri V, Stewart L. Extracting summary statistics to perform meta-analyses of the published literature for survival endpoints. Stat Med 1998;17:2815-34.

20. Guyatt GH, Oxman AD, Montori V, et al. GRADE guidelines: 5 . Rating the quality of evidence--publication bias. J Clin Epidemiol 2011;64:1277-82.

21. Guyatt GH, Cook DJ, Jaeschke R, et al. Grades of recommendation for antithrombotic agents: American College of Chest Physicians EvidenceBased Clinical Practice Guidelines (8th Edition). Chest 2008;133:123s-31s.

22. Tang W, Cao Z, Han M, et al. Hydroxychloroquine in patients with mainly mild to moderate coronavirus disease 2019: open label, randomised controlled trial. BMJ 2020;369:m1849. 
23. Rosenberg ES, Dufort EM, Udo T, et al. Association of treatment with hydroxychloroquine or azithromycin with in-hospital mortality in patients with COVID-19 in New York state. JAMA 2020;323:2493-502.

24. Boulware DR, Pullen MF, Bangdiwala AS, et al. A randomized trial of hydroxychloroquine as postexposure prophylaxis for Covid-19. N Engl J Med 2020;383:517-25.

25. Shippey EA, Wagler VD, Collamer AN. Hydroxychloroquine: an old drug with new relevance. Cleve Clin J Med 2018;85:459-67.

26. Touret F, de Lamballerie X. Of chloroquine and COVID-19. Antiviral Res 2020;177:104762.

27. Olofsson S, Kumlin U, Dimock K, et al. Avian influenza and sialic acid receptors: more than meets the eye? Lancet Infect Dis 2005;5:184-8.

28. Vincent MJ, Bergeron E, Benjannet S, et al. Chloroquine is a potent inhibitor of SARS coronavirus infection and spread. Virol J 2005;2:69.

29. Sapp JL, Alqarawi W, MacIntyre CJ, et al. Guidance on minimizing risk of drug-induced ventricular arrhythmia during treatment of COVID-19: a statement from the Canadian Heart Rhythm Society. Can J Cardiol 2020;36:948-51.

30. REUTERS. True claim: Brazilian chloroquine study on COVID-19 patients was halted after 11 deaths. 2020. Available online: https://jp.reuters.com/article/

Cite this article as: Ebina-Shibuya R, Namkoong H, Horita N, Kato H, Hara Y, Kobayashi N, Kaneko T. Hydroxychloroquine and chloroquine for treatment of coronavirus disease 19 (COVID-19): a systematic review and meta-analysis of randomized and non-randomized controlled trials. J Thorac Dis 2021;13(1):202-212. doi: 10.21037/jtd-20-2022 uk-factcheck-brazilian-chloroquine-study/trueclaimbrazilian-chloroquine-studyon-covid-19-patientswashalted-after11deaths-idUSKBN21Z395 (Accessed April 20, 2020).

31. Jorge A, Ung C, Young LH, et al. Hydroxychloroquine retinopathy - implications of research advances for rheumatology care. Nat Rev Rheumatol 2018;14:693-703.

32. Rainsford KD, Parke AL, Clifford-Rashotte M, et al. Therapy and pharmacological properties of hydroxychloroquine and chloroquine in treatment of systemic lupus erythematosus, rheumatoid arthritis and related diseases. Inflammopharmacology 2015;23:231-69.

33. Fanouriakis A, Kostopoulou M, Alunno A, et al. 2019 update of the EULAR recommendations for the management of systemic lupus erythematosus. Ann Rheum Dis 2019;78:736-45.

34. Rome BN, Avorn J. Drug Evaluation during the Covid-19 Pandemic. N Engl J Med 2020;382:2282-4.

35. Sackett DL, Rosenberg WMC, Gray JAM, et al. Evidence based medicine: What it is and what it isn't - It's about integrating individual clinical expertise and the best external evidence. Br Med J 1996;312:71-2.

36. Rana D, Dulal S. Therapeutic application of chloroquine and hydroxychloroquine in clinical trials for COVID-19: a systematic review. medRxiv 2020. doi: 10.1101/2020.03.22.20040964. 


\section{Appendix 1 Search formulas}

\section{PubMed}

(ncov OR covid OR 2019-nCoV OR COVID-19 OR (wuhan and coronavirus) OR sars-cov OR (2019 novel coronavirus)) AND (chloroquine or hydroxychloroquine)

\section{Web of Science}

TS = (ncov OR covid OR 2019-nCoV OR COVID-19 OR (wuhan and coronavirus) OR sars-cov OR (2019 novel coronavirus)) AND TS = (chloroquine or hydroxychloroquine)

\section{Embase}

(ncov OR covid OR '2019 ncov' OR 'covid 19'/exp OR 'covid 19' OR (wuhan AND ('coronavirus'/exp OR coronavirus)) OR 'sars cov'/exp OR 'sars cov' OR '2019 novel coronavirus'/exp OR '2019 novel coronavirus' OR (2019 AND novel AND ('coronavirus'/exp OR coronavirus))) AND ('chloroquine'/exp OR chloroquine OR 'hydroxychloroquine'/exp OR hydroxychloroquine)

\section{Cochrane}

\#1 ncov OR covid OR sars-cov OR novel coronavirus

\#2 chloroquine or hydroxychloroquine

$\# 3 \# 1$ and \#2

\section{MedRxiv}

chloroquine or hydroxychloroquine

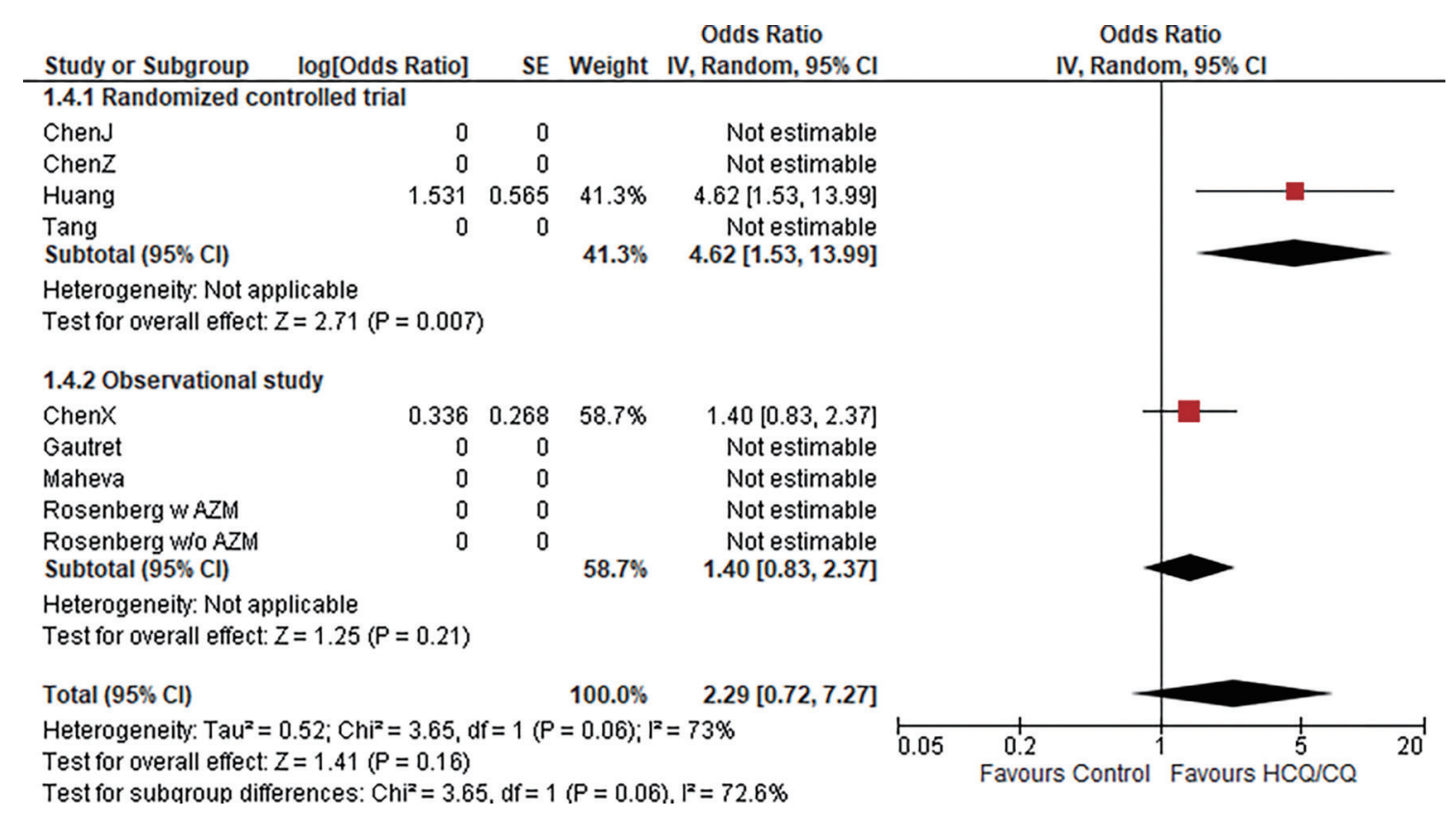

Figure S1 A forest plot for time to discharge. Rosenberg w AZM (with AZM): "HCQ + AZM versus AZM alone". Rosenberg w/o AZM (without AZM): "HCQ alone versus neither of them". SE, standard error; 95\% CI, 95\% confidence interval; IV, inverse variance; M-H, Mantel-Haenszel; HCQ/CQ, hydroxychloroquine/chloroquine; AZM, azithromycin. 


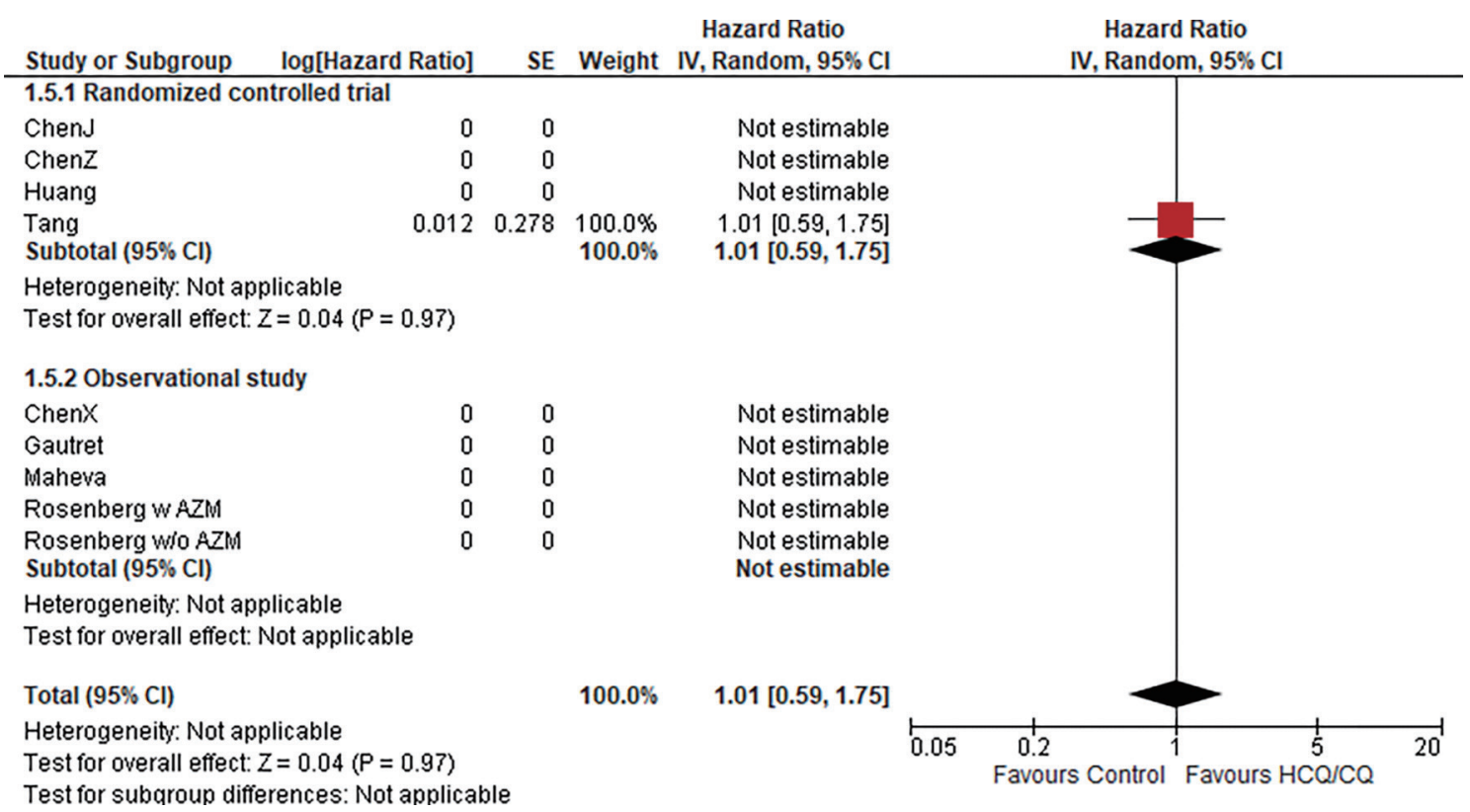

Figure S2 A forest plot for time to symptom alleviation. Rosenberg w AZM (with AZM): "HCQ + AZM versus AZM alone". Rosenberg w/ o AZM (without AZM): "HCQ alone versus neither of them". SE, standard error; 95\% CI, 95\% confidence interval; IV, inverse variance; M-H, Mantel-Haenszel; HCQ/CQ, hydroxychloroquine/chloroquine; AZM, azithromycin.

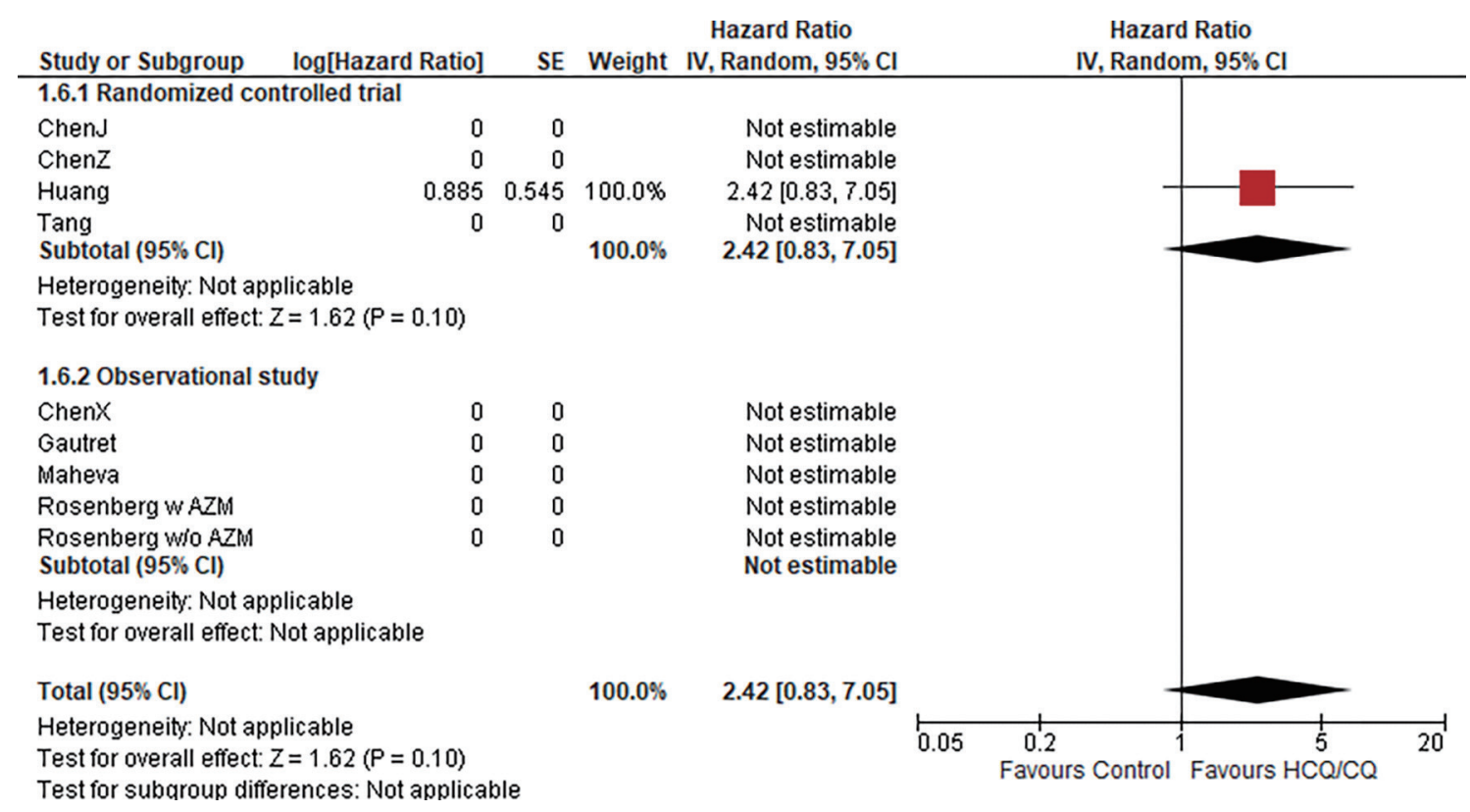

Figure S3 A forest plot for time to CT image improvement. Rosenberg w AZM (with AZM): "HCQ + AZM versus AZM alone". Rosenberg w/o AZM (without AZM): "HCQ alone versus neither of them". SE, standard error; 95\% CI, 95\% confidence interval; IV, inverse variance; M-H, Mantel-Haenszel; HCQ/CQ, hydroxychloroquine/chloroquine; AZM, azithromycin. 


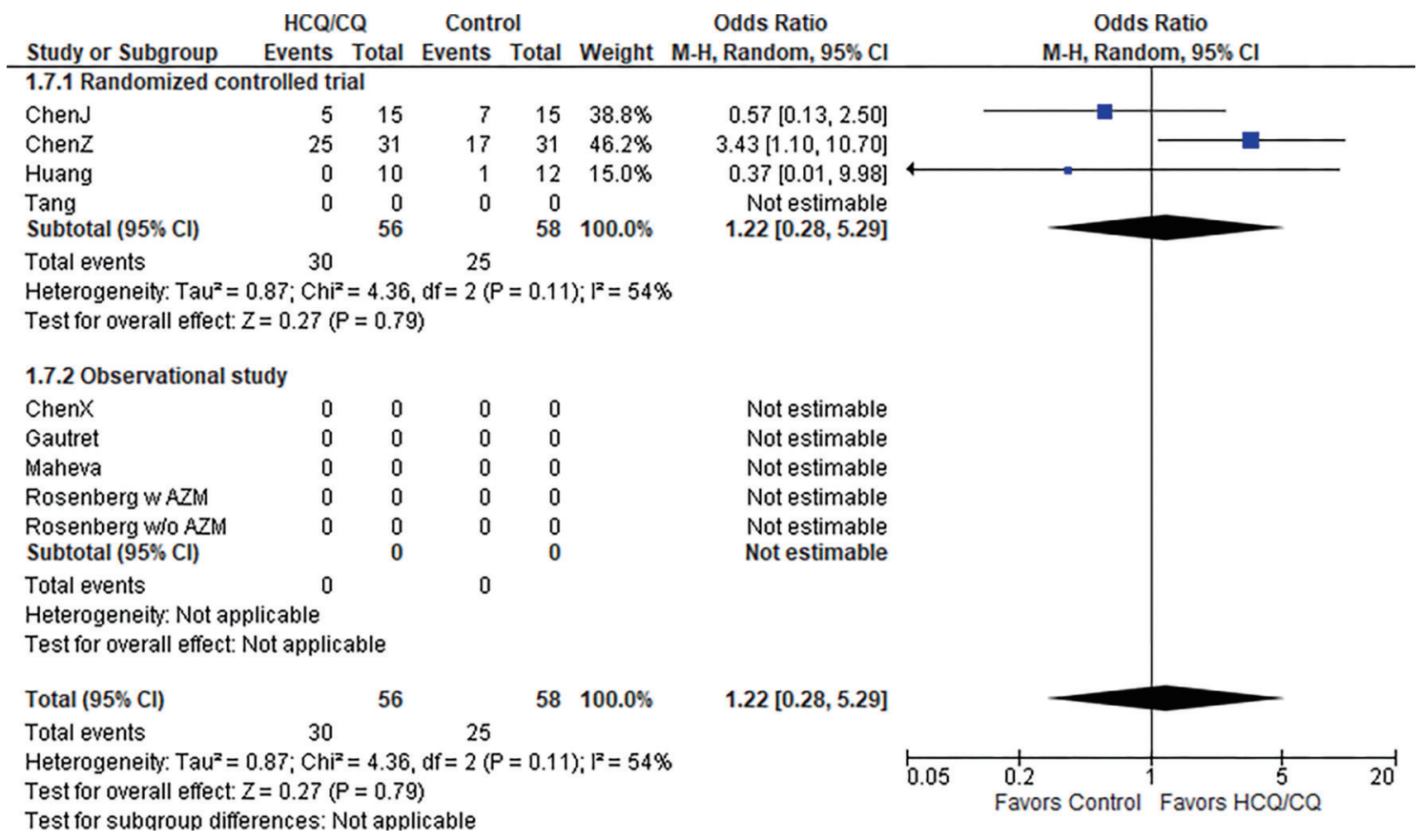

Figure S4 A forest plot for CT image improvement at day 7. Rosenberg w AZM (with AZM): "HCQ + AZM versus AZM alone". Rosenberg w/o AZM (without AZM): "HCQ alone versus neither of them". SE, standard error; 95\% CI, 95\% confidence interval; IV, inverse variance; M-H, Mantel-Haenszel; HCQ/CQ, hydroxychloroquine/chloroquine; AZM, azithromycin.

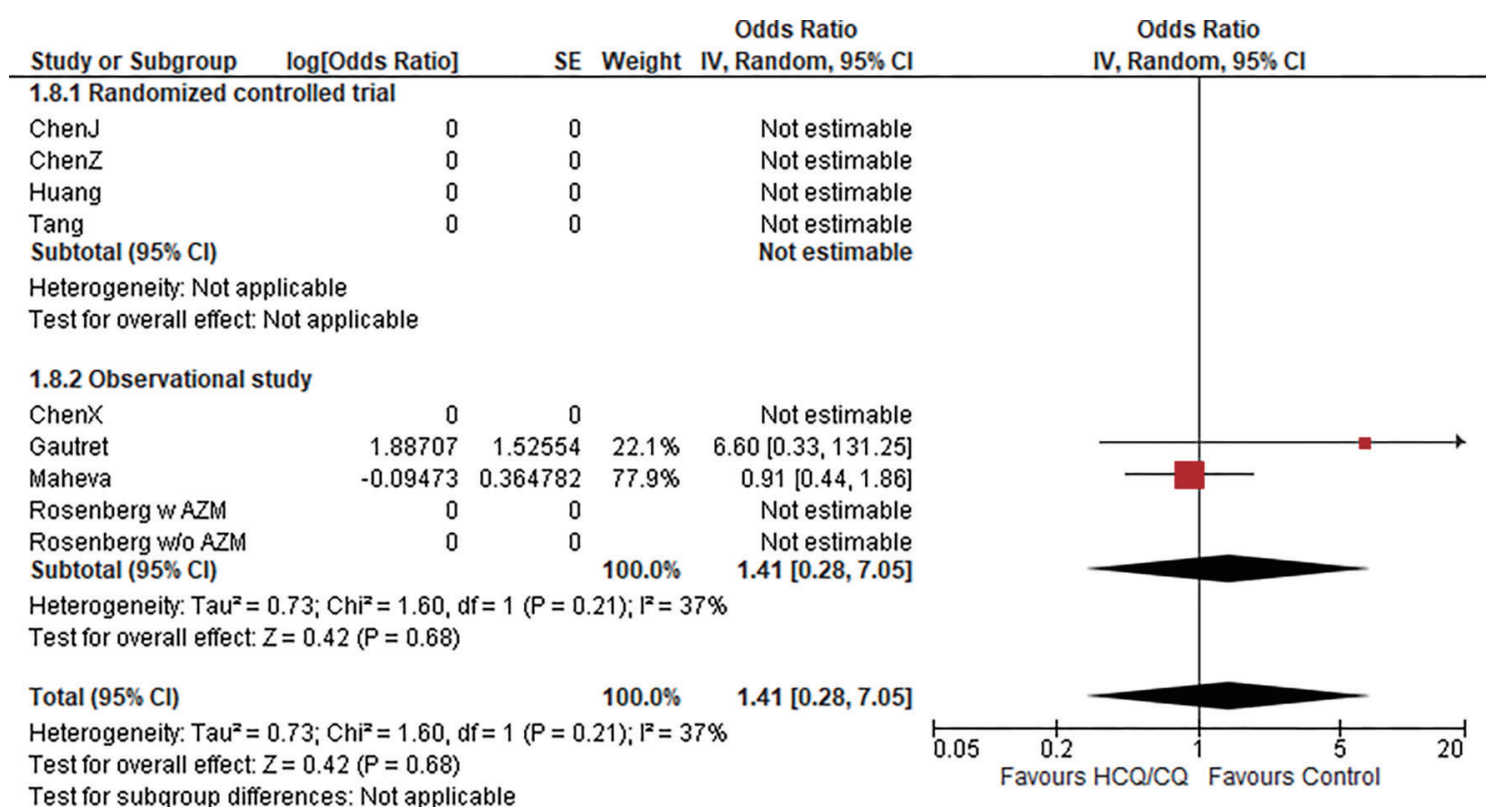

Figure S5 A forest plot for disease progression (death or intensive care unit admission). Rosenberg w AZM (with AZM): "HCQ + AZM versus AZM alone". Rosenberg w/o AZM (without AZM): "HCQ alone versus neither of them". SE, standard error; 95\% CI, 95\% confidence interval; IV, inverse variance; M-H, Mantel-Haenszel; HCQ/CQ, hydroxychloroquine/chloroquine; AZM, azithromycin. 
Odds Ratio

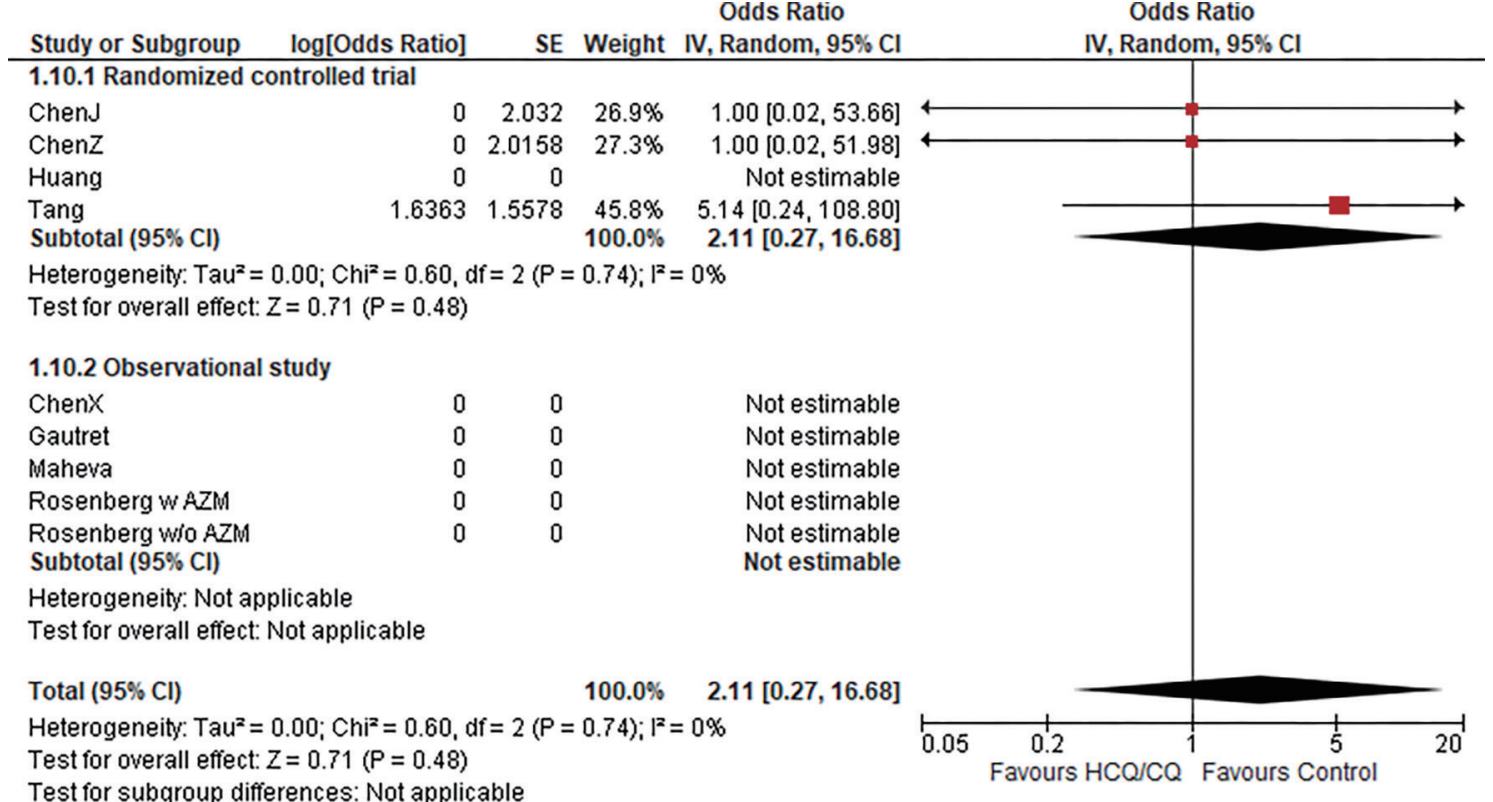

Figure S6 A forest plot for serious adverse event. Rosenberg w AZM (with AZM): "HCQ + AZM versus AZM alone". Rosenberg w/o AZM (without AZM): "HCQ alone versus neither of them". SE, standard error; 95\% CI, 95\% confidence interval; IV, inverse variance; M-H,

Mantel-Haenszel; HCQ/CQ, hydroxychloroquine/chloroquine; AZM, azithromycin.

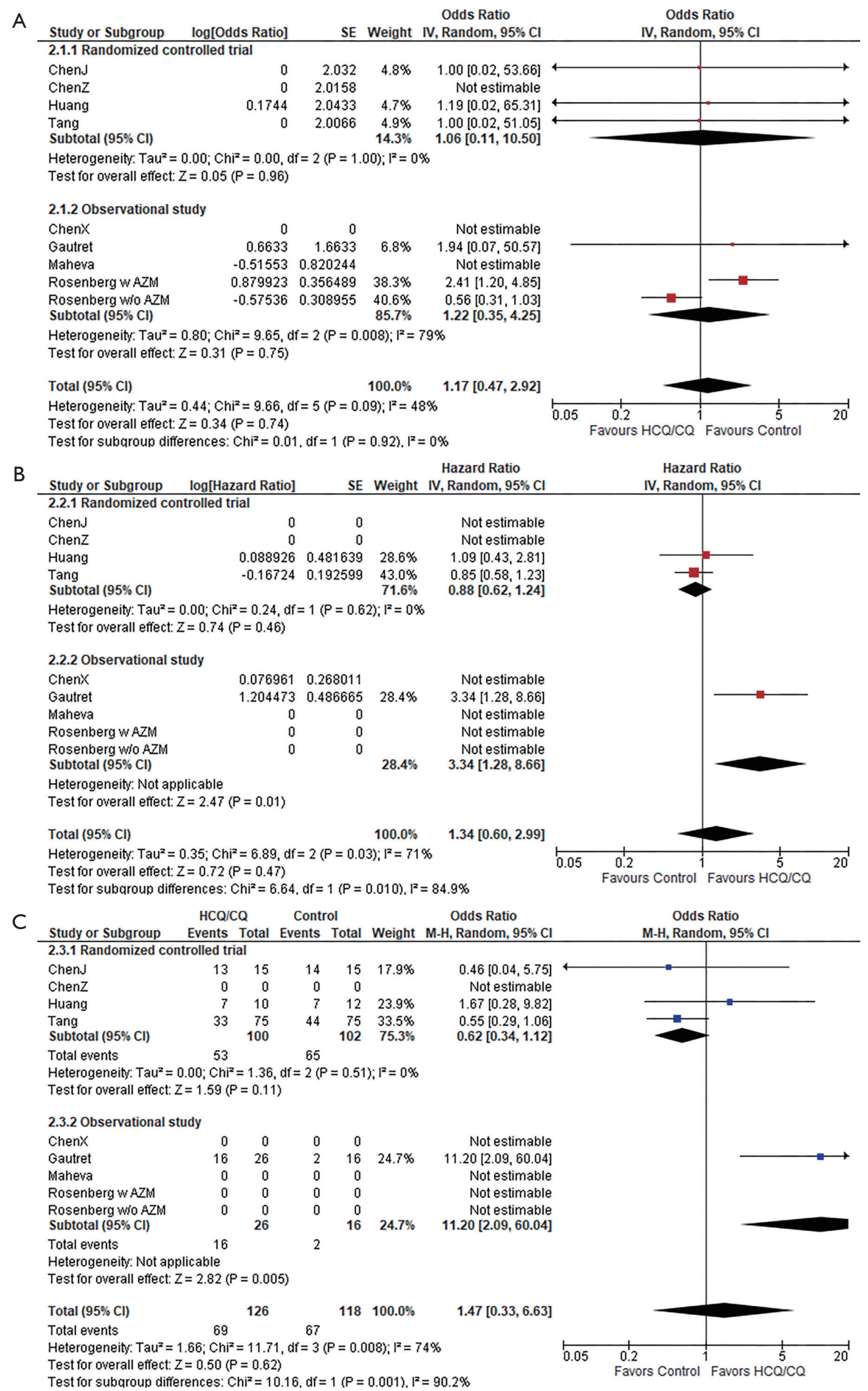

Figure S7 A forest plot for primary outcomes excluding non-peer reviewed articles. (A) All cause death; (B) time to viral clearance; (C) viral clearance at day 7. Rosenberg w AZM (with AZM): "HCQ + AZM versus AZM alone". Rosenberg w/o AZM (without AZM): "HCQ alone versus neither of them”. SE, standard error; $95 \%$ CI, 95\% confidence interval; IV, inverse variance; M-H, Mantel-Haenszel; HCQ/CQ, hydroxychloroquine/chloroquine; AZM, azithromycin. 